\title{
Electron-state tuning of multilayer graphene by defects
}

\author{
Ken Kishimoto* and Susumu Okada ${ }^{\dagger}$ \\ Graduate School of Pure and Applied Sciences, University of Tsukuba, Tsukuba, Ibaraki 305-8571, Japan
}

\begin{abstract}
Electronic band structures of graphene thin films, in which the topmost layer possesses atomic and topological defects, are studied using the density functional theory. Our calculations showed that all graphene thin films studied here have a finite energy gap in their $\pi$ electron states, although the thin films possess defect-free hexagonal networks, because of the spatially undulated local potential on pristine graphene layers induced by the defects. The energy gap in $\pi$ states slightly decreases with increasing number of layers, while the gap sensitively depends on the interlayer stacking arrangement. Our analyses clarify that the interlayer interaction plays a crucial role for mediating the effect of the defects on the $\pi$ electrons of pristine layers.
\end{abstract}

\section{Introduction}

The exfoliation of graphite ${ }^{1,2)}$ and thermal annealing of $\mathrm{SiC}{ }^{3,4)}$ have resulted in the single-layered graphite, graphene, which provides us an ultimate two-dimensional electronic system. Much effort has been devoted not only to elucidating the fundamental physics of this carbon allotrope but also to exploring the possibility of application in next-generation electronic devices . ${ }^{1,5-8)}$ Graphene possesses a pair of linear dispersion bands at the Fermi level resulting in a remarkable carrier mobility of a few hundred thousand $\mathrm{cm}^{2} / \mathrm{Vs}$ and the application of graphene in high-speed switching devices. However, these remarkable electronic properties of graphene are fragile against external conditions such as structural corrugation, atom/molecule absorption, and interactions with other graphene or substrates. ${ }^{7-15)}$ Moreover, for the switching device application, it is mandatory to generate the finite energy gap between conduction and valence bands, instead of the linear bands at the Fermi level. Although these issues cause difficulty in applying graphene to switching devices, graphene still is a fascinating material for semiconductor electronics devices. Thus, it is urgent us to show the possible procedure to control the electronic energy band of graphene without the modulation of their perfect hexagonal C-C network.

\footnotetext{
*E-mail: kkishimoto@comas.frsc.tsukuba.ac.jp

${ }^{\dagger}$ E-mail: sokada@comas.frsc.tsukuba.ac.jp
} 
It has been reported that the interlayer interaction ${ }^{16-20)}$ and defects $^{21-26)}$ substantially modulate the electronic structure of graphene from metallic to semiconducting or magnetic. These facts lead to an idea that allows us to tune the electronic structures of graphene thin films by controlling the stacking arrangement and defect species implanted. Indeed, in our previous work, ${ }^{27)}$ we demonstrated that bilayer graphene possesses a finite energy gap in the $\pi$ states upon introducing defects in one of the two layers. The gap in the $\pi$ states of the bilayer graphene sensitively depends on the defect species and their mutual stacking arrangement. The gap is ascribed to the cooperation between the spatially undulated electrostatic potential on a pristine layer caused by the defects and the interlayer interaction.

In this work, we aim to give further theoretical insight into the effect of atomic and topological defects on the electronic structures of multilayer graphene, and to show the possible procedure to tune the band structures of graphene thin films by controlling the defect species and interlayer interaction, within the framework of the density functional theory. Our calculations show that the energy gap of the bilayer graphene monotonically decreases with increasing interlayer spacing. However, on the other hand, the gap slightly decreases with increasing number of layers. We also found that the thin films with the rhombohedral (ABC) stacking have considerably larger gaps than do those with the hexagonal (AB) stacking, indicating that the interlayer hybridization plays a crucial role in controlling the electronic structures of graphene thin films, one of which layer possesses defects. Our findings lead to a possible procedure for tuning the electronic structures of graphene thin films by chemical and physical modifications of the outermost layer of the thin films.

\section{Calculation methods and structural model}

All calculations were performed within the framework of the density functional theory $^{28,29)}$ using the Simulation Tool for Atom Technology (STATE) package. ${ }^{30)}$ We used the local density approximation ${ }^{31,32)}$ to describe the exchange-correlation potential among the interacting electrons. An ultrasoft pseudopotential generated by the Vanderbilt scheme was used to describe the interaction between electrons and ions. ${ }^{33)}$ The valence wave functions and charge density were expanded in terms of the plane-wave basis set with cutoff energies of 25 and 225 Ry, respectively. To exclude unphysical dipole interaction with periodic images normal to the graphene layers within the conventional plane-wave basis-set scheme, we imposed an open boundary condition normal 
(a)

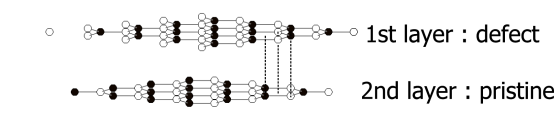

(c) (b)

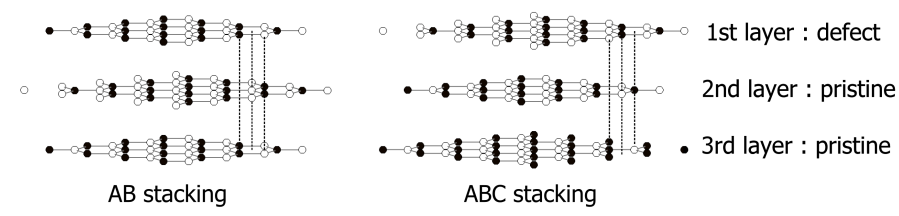

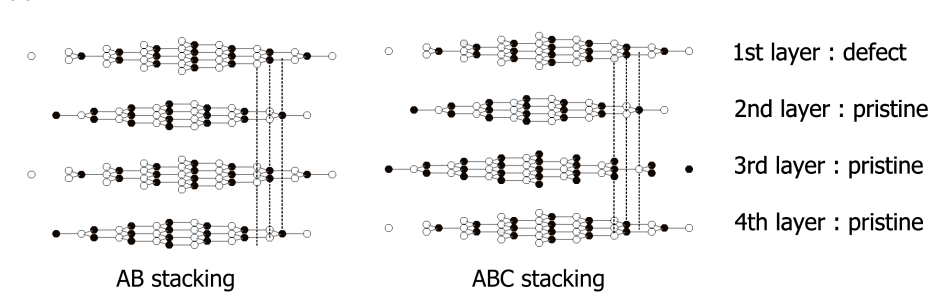

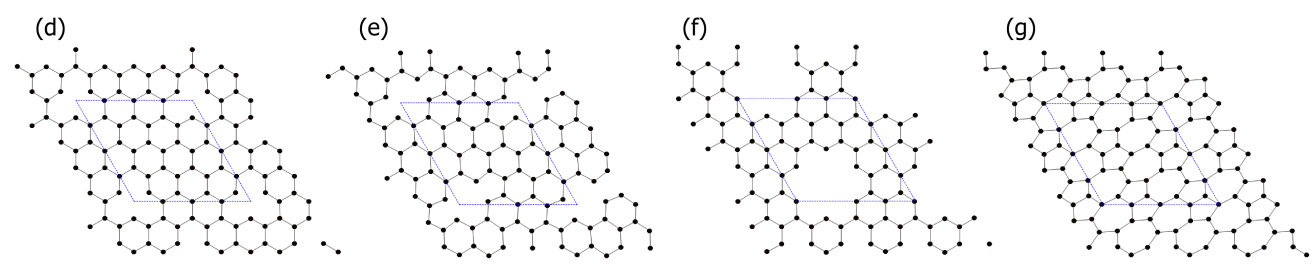

Fig. 1. (Color online) Geometric structures of (a) bilayer, (b) trilayer, and (c) tetralayer graphene thin films with the hexagonal and rhombohedral stacking arrangements, in which the topmost layer possesses (d) MV, (e) DV, (f) V6, and (g) SW. Blue dotted parallelograms denote the lateral unit cell.

to the layers by the effective screening medium (ESM) method..$^{34)}$

In the present work, we consider bilayer, trilayer, and tetralayer graphene thin films in which the topmost layer possesses atomic and topological defects (Fig. 1). For the defects, we consider monovacancy (MV), divacancy (DV), hexagonal vacancy (V6), and Stone-Wales (SW) defects per $4 \times 4$ lateral periodicity of graphene. Although the lateral lattice parameter strongly depends on the defect species and densities, we fixed the lateral parameter as $0.98 \mathrm{~nm}$, which corresponds to that of a $4 \times 4$ lateral cell of pristine graphene, to satisfy the commensurability condition between pristine and defective layers. For the bilayer graphene, we consider the hexagonal stacking arrangement under various interlayer spacings from 0.34 to $0.55 \mathrm{~nm}$ to clarify how the interlayer spacing affects the electronic structure of bilayer graphene, one of which layers possesses the defects. For the trilayer and tetralayer graphene thin films, we consider both hexagonal and rhombohedral stacking arrangements to clarify the effect of the stacking arrangement on the $\pi$ gap of the graphene thin films. During calculations for the trilayer and tetralayer graphene, we fixed the interlayer spacing at $0.34 \mathrm{~nm}$. Atomic structures of graphene and graphene with defects are optimized under the lateral cell of $0.98 \mathrm{~nm}$ until 
the force acting on each atom is less than $1.33 \times 10^{-3} \mathrm{HR} / \mathrm{au}$. Integration over the Brillouin zone was carried out using an equidistance mesh of $4 \times 4 \times 1 \boldsymbol{k}$ points for graphene thin films with $4 \times 4$ lateral periodicity, which correspond to the $16 \times 16 \times 1 \boldsymbol{k}$-point grid for the primitive cell of graphene, resulting in sufficient convergence in their electronic structures.

\section{Results and discussion}

Figure 2(a) shows the energy gap of the bilayer graphene with defects as a function of the interlayer spacing. For the interlayer spacing of $0.34 \mathrm{~nm}$, the $\pi$-states energy gaps are 336, 67, 318, and $147 \mathrm{meV}$ for the bilayer graphene with monovacancies, divacancies, hexagonal vacancies, and topological defects, respectively, because of the undulation of the electrostatic potential on the pristine layer caused by the defects. As shown in Figs. 2(b)-2(e), the electrostatic potential has a convex/concave with height ranging from 35 to $434 \mathrm{meV}$ depending on the defects. The gap monotonically decreases with increasing interlayer spacing and finally vanishes at critical interlayer distances. The critical interlayer distance strongly depends on the defect species. The critical distances are $0.53,0.47,0.55$, and $0.50 \mathrm{~nm}$ for the monovacancy, divacancy, hexagonal vacancy, and topological defects, respectively. Under the interlayer distance of $0.40 \mathrm{~nm}$, electrostatic potential undulation above the pristine layer is smaller than that under the distance of $0.34 \mathrm{~nm}$ [Figs. 2(b)-2(i)].

Figures 3(a) and 3(b) show the electronic structures of the trilayer graphene with the hexagonal and rhombohedral stacking arrangements, in which the topmost layer possesses monovacancies. For both stacking arrangements, two dispersive bands and three flat dispersion bands appear near the Fermi level. Two of three flat dispersion bands are strongly localized at the defect sites and exhibit a dangling bond nature associated with the $\sigma$ state at the defects. The remaining state exhibits a hybridized nature between $\pi$ and the dangling bond $\sigma$ states. Thus, we classify these three states as the states associated with the defect. In contrast, two dispersive bands have the $\pi$ nature distributed not only on the pristine but also on the defective layers. These two dispersive bands still have finite energy gaps of 13 and $327 \mathrm{meV}$ for the hexagonal and rhombohedral stacking thin films, respectively. Thus, the gap in $\pi$ states strongly depends on the stacking arrangement, indicating that the hybridization of the wave function between layers plays an important role in determining the electronic structure modulation of graphene thin films with defects. As stated above, the three less dispersive 


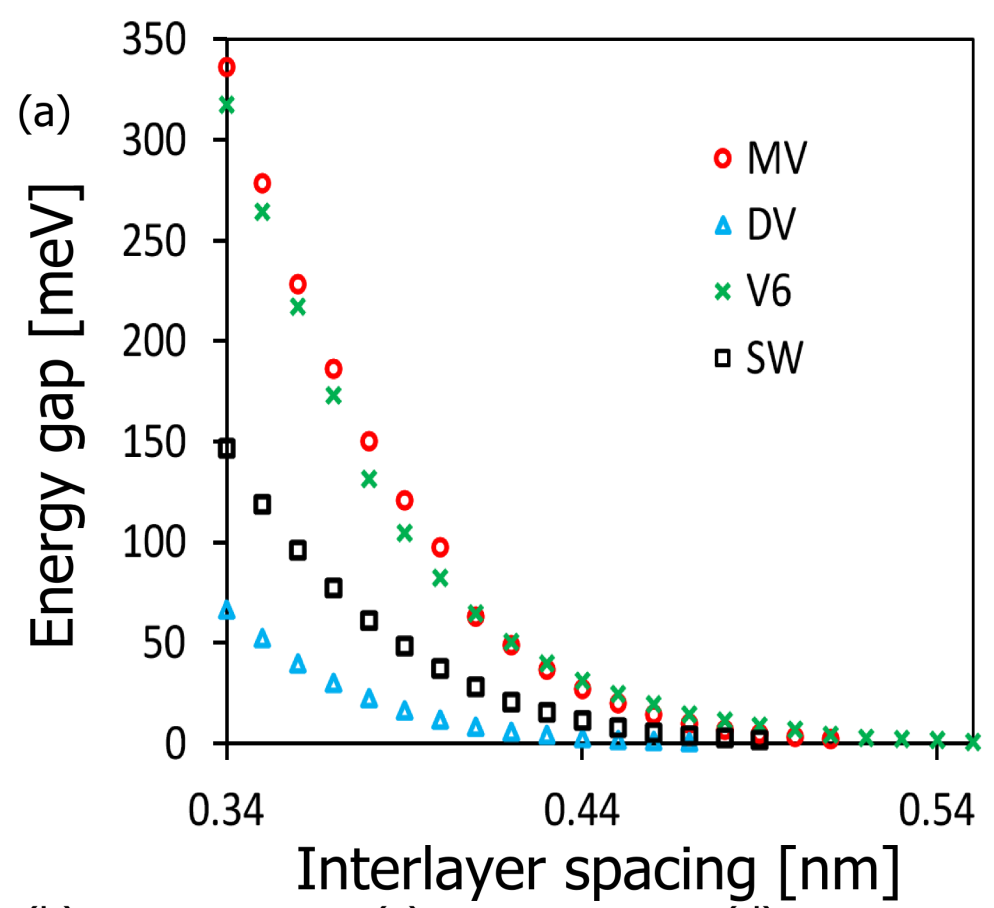

(b)

(c)

(d)

(e)

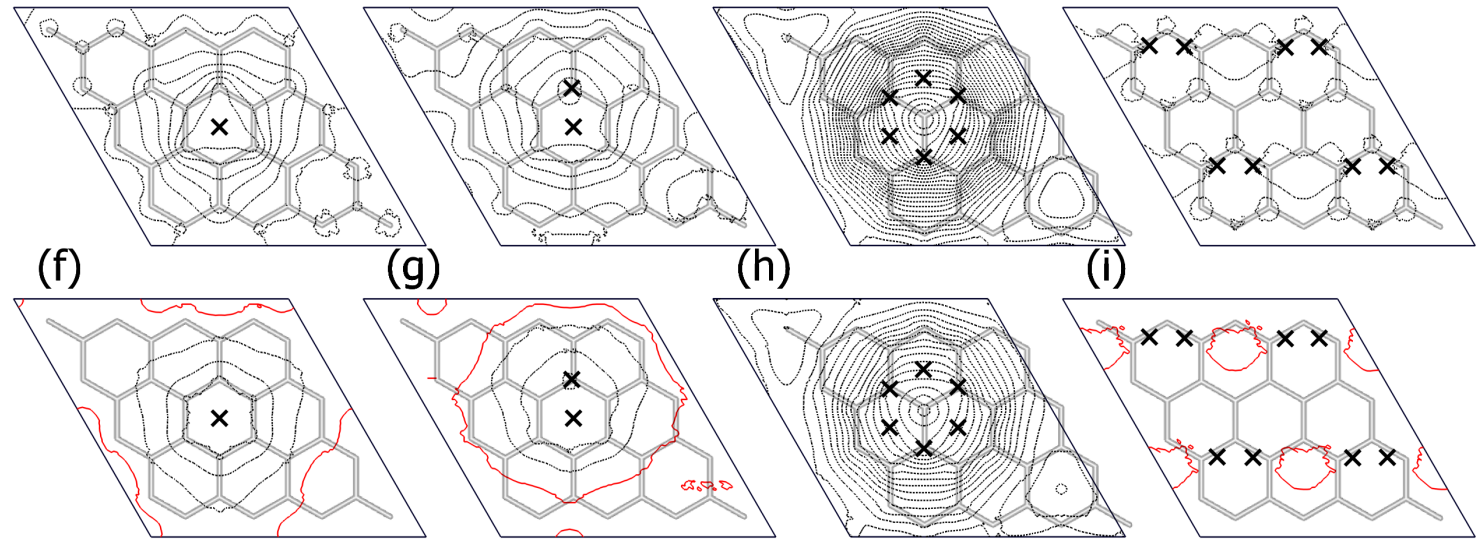

Fig. 2. (Color online) (a) Energy gap in $\pi$ states of the bilayer graphene with defects as a function of the interlayer spacing. Circles, triangles, crosses, and squares denote the gap in the bilayer graphene with MV, DV, V6, and SW defects, respectively. Contour plots of electrostatic potential modulation on the pristine layer of bilayer graphene with (b) MV, (c) DV, (d) V6, and (e) SW defects under the interlayer spacing of $0.34 \mathrm{~nm}$. Contour plots of electrostatic potential modulation on the pristine layer of bilayer graphene with (f) MV, (g) DV, (h) V6, and (i) SW defects under the interlayer spacing of $0.40 \mathrm{~nm}$. Solid and dotted lines denote regions where the potential increases and decreases from that of the isolated graphene without defects, respectively. Each contour represents the potential difference of $0.0006 \mathrm{HR}$. Crosses denote the position of the atomic and topological defects in the defective graphene. The $\mathrm{sp}^{2}$ network of the pristine graphene layer is denoted by the pale cylinders. 

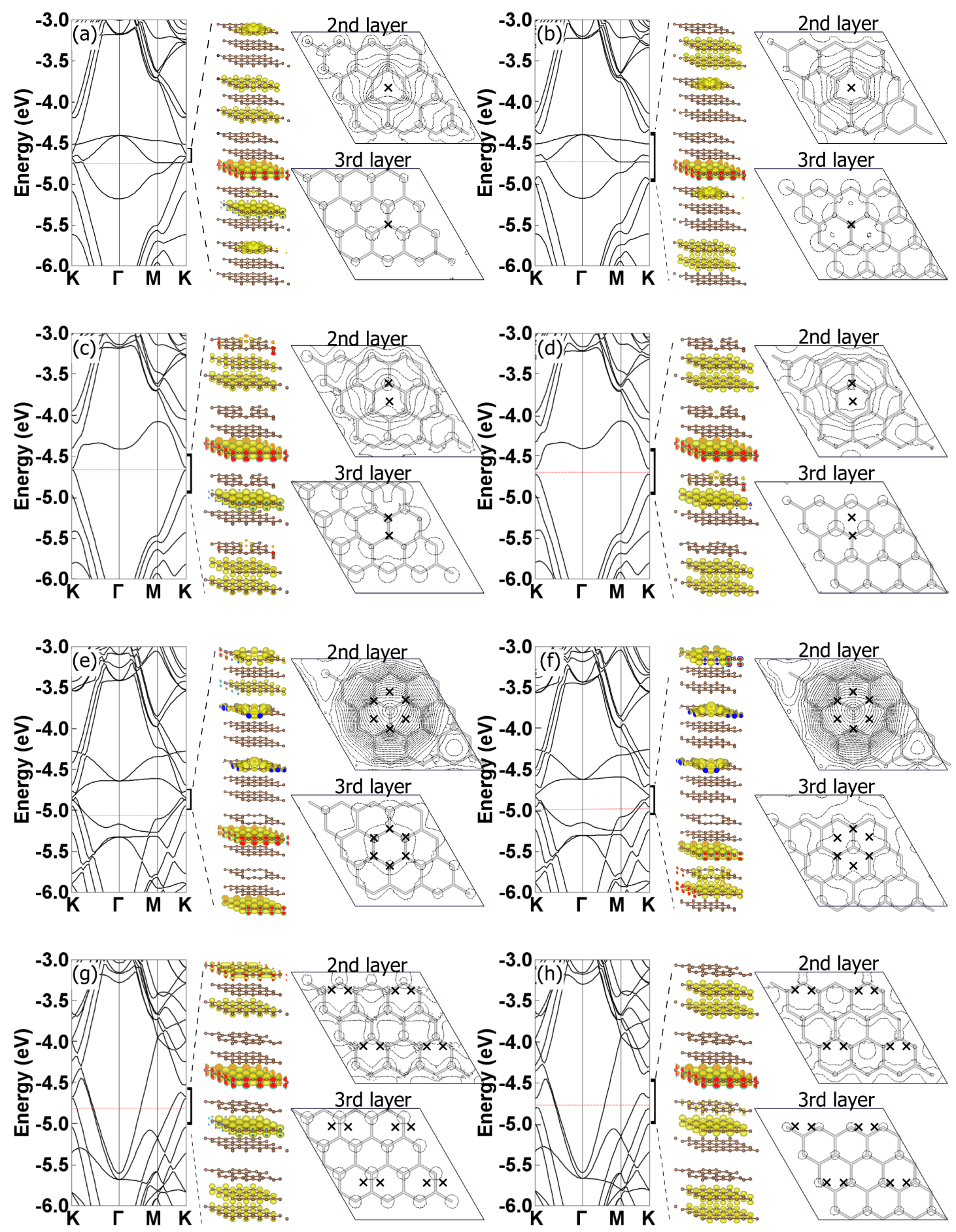

Fig. 3. (Color online) Electronic structures of trilayer graphene with (a) hexagonal (AB) stacking and MV, (b) rhombohedral (ABC) stacking and MV, (c) AB stacking and DV, (d) ABC stacking and DV, (e) AB stacking and V6, (f) ABC stacking and V6, (g) AB stacking and SW, and (h) ABC stacking and SW. Energies are measured from that of the vacuum level. The dotted horizontal line denotes the Fermi level. In each figure, center and right panels show the isosurfaces of wave functions near the Fermi level at the $\mathrm{K}$ point and the contour plots of potential modulations above the pristine layers owing to the adsorption of the defective layer, respectively. Each contour corresponds to the potential difference of $0.0006 \mathrm{HR}$. Crosses in the contour plots denote the positions of the defects. Solid and dotted lines denote regions where the potential increases and decreases from that of the isolated graphene without defects, respectively. 
bands are localized on the defects, so that the states are not expected to contribute to electron transfer through the thin films. Therefore, the thin films with monovacancies may exhibit a semiconducting transport property by tuning the Fermi level, although they still contain perfect hexagonal networks.

By focusing on the electrostatic potential on each atomic layer of trilayer graphene with monovacancies, we find that the interlayer stacking arrangement also affects the potential on the pristine layers: The potential undulation in the pristine layer in the trilayer graphene with the hexagonal stacking is smaller than that with the rhombohedral stacking. Furthermore, we observe substantial potential undulation on the third layer of the thin films which is separated by $0.68 \mathrm{~nm}$ from the defective graphene layer. Therefore, the interlayer interaction mediates the potential modulation due to the monovacancy.

In the cases of trilayer graphene with divacancies, the thin films are semiconductors with the finite energy gap between valence and conduction bands [Figs. 3(c) and 3(d)]. The calculated energy gaps are 19 and $71 \mathrm{meV}$ for the hexagonal and rhombohedral stacking arrangements, respectively. In these cases, four electron states emerge near the Fermi level around the $\mathrm{K}$ point. These four states exhibit the $\pi$ nature and are primarily extended throughout the pristine layers, as shown in the middle panel of Figs. 3(c) and 3(d). In these cases, we also observe electrostatic potential modulation on each atomic layer. The potential modulation occurs not only on the second but also on the bottommost layers. In contrast to the trilayer graphene with monovacancies, the potential undulation on the bottommost layer of the film with the rhombohedral stacking is smaller than that in the case of the hexagonal stacking.

In the case of hexagonal vacancies [Figs. 3(e) and 3(f)], the trilayer graphene is a metal in which two dispersive and one flat band cross the Fermi level. Near the Fermi level and around the $\mathrm{K}$ point, three dispersive bands associated with the $\pi$ states still have finite energy gaps of 62 and $378 \mathrm{meV}$ for the hexagonal and rhombohedral stacking arrangements, respectively. The wave function analysis revealed that the remaining two are localized at the edge of the defect possessing the dangling bond nature of the unsaturated $\sigma$ state. In addition to the electronic structure modulation, the potential undulation still occurs on the third layer. In this case, we find the deep electrostatic potential valley below the vacancy in the second and bottommost graphene layers.

In addition to the atomic vacancies, the topological defect also affects the electronic structure of graphene thin films. Figures $3(\mathrm{~g})$ and $3(\mathrm{~h})$ show the electronic structures 
of the trilayer graphene with the hexagonal and rhombohedral stacking arrangements, respectively, in which the topmost layer possesses topological defects. Both thin films are metals in which several energy bands cross the Fermi level. Although the electron states associated with the $\sigma$ bond are absent, electron states near the Fermi level do not exhibit linear dispersion but have quadric dispersion leading to the finite energy gap between these states. The wave function of the states are distributed on the pristine layer [middle panel of Figs. 3(g) and 3(h)]. The calculated gaps in $\pi$ states are 14 and $132 \mathrm{meV}$ for the hexagonal and the rhombohedral stacking. For the electrostatic potential on each atomic layer, the potential undulation on the second layer is smaller than that in the other thin films with atomic defects. Furthermore, negligible potential undulation is observed on the bottommost layer for the thin films with the hexagonal and the rhombohedral stacking.

Table I. Energy gap in $\pi$ states of trilayer and tetralayer graphene thin films with hexagonal $(\mathrm{H})$ and rhombohedral $(\mathrm{R})$ interlayer stacking.

\begin{tabular}{ccccc}
\hline & \multicolumn{3}{c}{ Trilayer $(\mathrm{meV})$} & \multicolumn{2}{c}{ Tetralayer $(\mathrm{meV})$} \\
& $\mathrm{H}$ & $\mathrm{R}$ & $\mathrm{H}$ & $\mathrm{R}$ \\
\hline Monovacancy & 13 & 327 & 15 & 318 \\
Divacancy & 19 & 71 & 4 & 80 \\
Hexagonal vacancy & 62 & 378 & 14 & 295 \\
Topological defect & 14 & 132 & 8 & 111 \\
\hline
\end{tabular}

With increasing number of layers, the tetralayer graphene thin films, in which the topmost layer possesses the defects, still have finite energy gaps in $\pi$ states on pristine layers depending on defect species and stacking arrangement. Table I summarizes the energy gaps in $\pi$ states of pristine layers for the trilayer and tetralayer graphene thin films with the hexagonal and rhombohedral stacking arrangements, in which the topmost layer possesses monovacancies, divacancies, hexagonal vacancies, or topological defects. The gaps slightly decrease with increasing number of layers, except for the graphene thin films with monovacancies and divacancies. We also found that the gaps strongly depend on the interlayer stacking arrangements of graphene layers. For trilayer and tetralayer thin films, the thin films with the rhombohedral stacking have wider gaps in their $\pi$ states compared with the thin films with the hexagonal stacking. The finite energy gap in $\pi$ states still emerges not only for the trilayer but also in the 
tetralayer graphene thin films, although the thicknesses of these films are greater than the critical spacing of gap vanishing for bilayer graphene with defects. These findings corroborate the idea that the influence of defects is mediated by the orbital hybridization between graphene layers. In the case of graphene thin films with the rhombohedral stacking arrangement, all atoms belonging into inner layers have their counterparts in the adjacent atomic layers, in sharp contrast to the case of hexagonal stacking in which one of two atoms in the unit cell does not have its counterpart in adjacent layers (Fig. 1). In accordance with the stacking sequence, in the rhombohedral stacking, $\pi$ states of all $\mathrm{C}$ atoms hybridize with the $\pi$ states of $\mathrm{C}$ atoms in the adjacent layers, leading to the percolation of $\pi$ states throughout the layers.

\section{Summary}

We studied the electronic structures of few-layer graphene, in which the topmost layer possesses atomic or topological defects, using the density functional theory with local density approximation. Our calculations showed that the energy gap of bilayer graphene monotonically decreases with increasing interlayer spacing and finally vanishes at the critical interlayer spacing of about $0.50 \mathrm{~nm}$ depending on the defect species. For the trilayer and tetralayer graphene in which the topmost layer possesses atomic and topological defects, we found that they have a finite energy gap in the $\pi$ state, although the number of pristine layers increases and the thickness of the thin films is greater than the critical spacing of gap vanishing in the bilayer graphene with the defects. Moreover, we also found that the gap in such multilayer graphene with defects strongly depends on the interlayer stacking arrangement. For all atomic and topological defects studied here, the thin films with the rhombohedral stacking arrangement possess larger energy gaps in $\pi$ states than those in the case of the hexagonal stacking arrangement. The findings imply that the interlayer interaction plays an important role in mediating the effect of the defects that cause the potential undulation on the pristine layers. Our findings provide a hint to a possible procedure for realizing semiconducting graphene thin films that retain perfect graphitic layers except the outermost defective layers, allowing us to utilize such graphene thin films as the semiconductor electronic devices.

\section{Acknowledgments}

This work was supported in part by a Grant-in-Aid for Scientific Research from the Ministry of Education, Culture, Sports, Science and Technology of Japan and the Joint 
Research Program on Zero-Emission Energy Research, Institute of Advanced Energy, Kyoto University. Computations were performed on NEC SX-8/4B at the University of Tsukuba, SGI ICE XA/UV at the Institute for Solid State Physics, The University of Tokyo, and NEC SX-Ace at the Cybermedia Center, Osaka University. 


\section{References}

1) K. S. Novoselov, A. K. Geim, S. V. Morozov, D. Jiang, Y. Zhang, S. V. Dubonos, I. V. Grigorieva, and A. A. Firsov, Science 306, 666 (2004).

2) A. K. Geim and K. S. Novoselov, Nat. Mater. 6, 183 (2007).

3) I. Forbeaux, J.-M. Themlin, and J.-M. Debever, Phys. Rev. B 58, 16396 (1998).

4) C. Berger, Z. Song, X. Li, X. Wu, N. Brown, C. Naud, D. Mayou, T. Li, J. Hass, A. N. Marchenkov, E. H. Conrad, P. N. First, and W. A. de Heer, Science 312, 1191 (2006).

5) K. S. Novoselov, A. K. Geim, S. V. Morozov, D. Jiang, M. I. Katsnelson, I. V. Grigorieva, S. V. Dubonos, and A. A. Firsov, Nature 438, 197 (2005).

6) Y. Zhang, Y.-W. Tan, H. L. Stormer, and P. Kim, Nature 438, 201 (2005).

7) J. B. Oostinga, H. B. Heersche, X. Liu, A. F. Morpurgo, and L. M. K. Vandersypen, Nat. Mater. 7, 151 (2007).

8) Y. Zhang, T. Tang, C. Girit, Z. Hao, M. C. Martin, A. Zettl, M. F. Crommie, Y. R. Shen, and F. Wang, Nature 459, 820 (2009).

9) M. F. Craciun, S. Russo, M. Yamamoto, J. B. Oostinga, A. F. Morpurgo, and S. Tarucha, Nat. Nanotechnol. 4, 383 (2009).

10) T. Ohta, A. Bostwick, T. Seyller, K. Horn, and E. Rotenberg, Science 313, 951 (2006).

11) S. Y. Zhou, G.-H. Gweon, A. V. Fedorov, P. N. First, W. A. de Heer, D.-H. Lee, F. Guinea, A. H. C. Neto, and A. Lanzara, Nat. Mater. 6, 770 (2007).

12) A. Mattausch and O. Pankratov, Phys. Rev. Lett. 99, 076802 (2007).

13) N. T. Cuong, M. Otani, and S. Okada, Phys. Rev. Lett. 106, 106801 (2011).

14) K. Kamiya, N. Umezawa, and S. Okada, Phys. Rev. B 83, 153413 (2011).

15) S. Okada and T Kawai, Jpn. J. App. Phys. 51, 02BN05 (2012).

16) S. Konabe and S. Okada, J. Phys. Soc. Jpn. 81, 113702 (2012).

17) M. Koshino and T. Ando, Phys. Rev. B 76, 085425 (2007).

18) M. Koshino and T. Ando, Phys. Rev. B 77, 115313 (2008).

19) M. Otani, M. Koshino, Y. Takagi, and S. Okada, Phys. Rev. B 81, 161403(R) (2010).

20) W. Bao, L. Jing, J. Velasco Jr, Y. Lee, G. Liu, D. Tran, B. Standley, M. Aykol, S. B. Cronin, D. Smirnov, M. Koshino, E. McCann, M. Bockrath, and C. N. Lau, Nat. Phys. 7, 948 (2011). 
21) Y. Ma, P. O. Lehtinen, A. S. Foster, and R. M. Nieminen, New J. Phys. 6, 68 (2004).

22) H. Amara, S. Latil, V. Meunier, Ph. Lambin, and J.-C. Charlier, Phys. Rev. B 76, 115423 (2007).

23) M. M. Ugeda, I. Brihuega, F. Hiebel, P, Mallent, J.-Y. Veuillen, J. M.

G.-Rodriguez, and F. Yndurain, Phys. Rev. B 85, 121402(R) (2012).

24) M. Dvorak, W. Oswald, and Z. Wu, Sci. Rep. 3, 2289 (2013).

25) P. Koskinen, S. Malola, and H. Häkkinen, Phys. Rev. Lett. 101, 115502 (2008).

26) S. Okada, T. Kawai, and K. Nakada, J. Phys. Soc. Jpn. 80, 013709 (2011).

27) K. Kishimoto and S. Okada, Surf. Sci. 644, 18 (2016).

28) P. Hohenberg and W. Kohn, Phys. Rev. 136, B864 (1964).

29) W. Kohn and L. J. Sham, Phys. Rev. 140, A1133 (1965).

30) Y. Morikawa, K. Iwata, and K. Terakura, Appl. Surf. Sci. 11, 169 (2000).

31) J. P. Perdew and A. Zunger, Phys. Rev. B 23, 5048 (1981).

32) D. M. Ceperley and B. J. Alder, Phys. Rev. Lett. 45, 566 (1980).

33) D. Vanderbilt, Phys. Rev. B 41, 7892 (1990).

34) M. Otani and O. Sugino, Phys. Rev. B 73, 115407 (2006). 